\title{
Single-Crystal Molybdenum Solar Thermal Propulsion Thruster
}

\author{
By Hironori Sahara, ${ }^{1)}$ Kai Watanabe, ${ }^{2)}$ Morio Shimizu $^{3)}$ and Yoshihiro NaKAmura ${ }^{2)}$ \\ 1) Japan Society for the Promotion of Science, Tokyo, Japan \\ ${ }^{2)}$ Faculty of Engineering, Hosei University, Koganei, Japan \\ ${ }^{3)}$ National Aerospace Laboratory of Japan, Chofu, Japan
}

(Received October 29th, 2002)

\begin{abstract}
We detail the design, fabrication, and experimental and calculation results for three cavity solar thermal propulsion (STP) thrusters - medium, small, and very small - made of single-crystal molybdenum (SC-Mo), developed in the National Aerospace Laboratory of Japan (NAL). We obtained very high temperatures - 2,300 K for the propellant gas and 2,200 K on the outer surface of the thruster - at an appropriate propellant flow with the small thruster by solar-heating with a suitable concentrator of $1.05 \mathrm{~m}$ in diameter, which corresponds to an 800 -second-class specific impulse thruster. Temperatures obtained in experiments were much lower than expected, however. To find points for improvement and evaluate thruster performance in space, we conducted a model calculation that confirmed the thruster could achieve an 800 to 900 -second-class specific impulse with a $0.1-2 \mathrm{~N}$ class thrust magnitude in space. STP is thus a candidate for near-future propulsion in applications such as upper stages of orbit transfer vehicles.
\end{abstract}

Key Words: Solar Thermal Propulsion Thruster, Single-Crystal Molybdenum, Solar Rays Concentrator, Orbit Transfer Vehicle, Upper Stage

\section{Introduction}

Due to its high thrust density, a potentially attractive propulsion concept offering significant payload gains for orbit transfer, from low-earth-orbit (LEO) to higher orbits such as station keeping and spacecraft attitude control, is thermal propulsion using a light gas as a propellant and different types of heat energy. Solar thermal propulsion (STP) has a high specific impulse $\left(I_{s p}\right)$ of 500-1,000 seconds in an appropriate thrust magnitude up to $10 \mathrm{~N}$ and potentially results in much less space pollution than conventional propulsion. This promising feature has led the United States Air Force (USAF) and National Aeronautics and Space Administration (NASA) to develop STP for orbit transfer vehicles (OTVs), doubling the geostationary orbit (GEO) mission payload capability. ${ }^{1-9)}$

A vital component in STP is the thruster, which is made of refractory metals such as tungsten, rhenium, tantalum, and molybdenum or advanced high-temperature ceramics because of the high propellant temperature involved - up to $3,000 \mathrm{~K} .{ }^{6,7)}$ The USAF is developing an integrated solar upper stage (ISUS) and integrated high payoff rocket propulsion technology (IHPRPT) for a solar orbit transfer vehicle (SOTV) of the Air Force Research Laboratory (AFRL). ${ }^{8,9)}$ Tungsten, used for the thruster in conventional SOTVs concepts and experiments, however, becomes brittle due to recrystallization at high temperatures, as has also been reported for a DC arcjet nozzle of pure tungsten after operation. ${ }^{10)}$ Rhenium, considered ideal for STP thrusters, costs considerably more than other refractory materials, which has hampered the development of rhenium-based STP manufactur-

(C) 2003 The Japan Society for Aeronautical and Space Sciences ing technology. This has led to the study of a tungsten alloy thruster with a heat storage container to maintain a high temperature and preventing recrystallization when the vehicle passes through the earth's shadow.

The National Aerospace Laboratory of Japan (NAL) began preliminary STP studies some time ago, for which the National Institute for Materials Science (NIMS) developed and patented single-crystal molybdenum (SC-Mo) and single-crystal tungsten $(\mathrm{SC}-\mathrm{W}) .{ }^{11,12)}$ These ideal materials provide high material strength and eliminate the possibility of brittleness due to recrystallization.

\section{SC-Mo NAL STP Thruster}

For the typical coaxial cavity STP thruster developed by the NAL (Fig. 1), solar rays concentrated via a concentrator

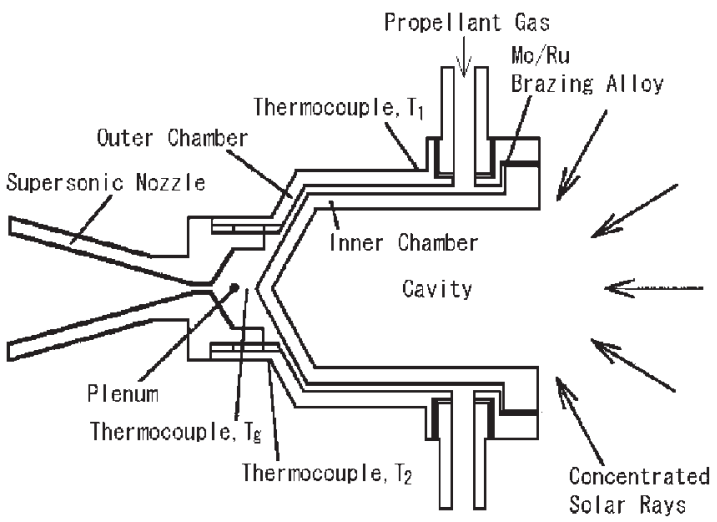

Fig. 1. Typical coaxial windowless NAL STP thruster, having a cavity for effective heating and double walls for propellant heating. Solar rays concentrated by a concentrator such as paraboloidal mirror or lens are input to the edge of the thruster and cavity from the right 
such as a paraboloidal mirror or lens are input to the edge of the thruster and the cavity from the right. The propellant gas is supplied through a pair of pipes and heated as it flows between outer and inner walls into a plenum where the propellant temperature is about $2,000 \mathrm{~K}$ and pressure is several atmospheres. It is exhausted through a nozzle as a supersonic flow to provide thrust. Thermal regeneration is possible because the thruster is supported by the pair of propellant supply pipes alone. In this process, the thruster temperature becomes very high, necessitating a refractory metal.

Body-centered cubic (b.c.c.) crystal refractory metals such as molybdenum and tungsten and their alloys have the advantages of a high melting point, strength at elevated temperatures, high thermal conductivity, low thermal expansion, and good resistance to liquid metal corrosion hence their wide industrial use. They do, however, lose critical ductility after recrystallization or welding, generally assumed to be due to intergranular embrittlement, which is the greatest weak point of these metals. NIMS et al. developed new technology for use in producing commercial-scale SCMo and SC-W from hot-rolled sheet doped with $\mathrm{CaO}$ and/or $\mathrm{MgO}$ by intentional secondary recrystallization. This made it possible to produce a SC-Mo sheet up to $20 \mathrm{~mm}$ thick, a SC-Mo cylinder nearly $30 \mathrm{~mm}$ in diameter, a SC-W sheet up to $3 \mathrm{~mm}$ thick, and a SC-W rod $8 \mathrm{~mm}$ in diameter. These are very promising for STP thruster applications, and NAL has manufactured SC-Mo and SC-W thrusters in several sizes. High-temperature Mo/Ru brazing — its melting point is about $2,300 \mathrm{~K}$, much higher than other brazing materials - and tungsten chemical vapor deposition (W-CVD) were used to seal the propellant gas in the thruster's joints. WCVD coating has the lowest vapor pressure among metals and prevents the molybdenum wall of the thruster from vaporizing at high temperature and vacuum. Mo/Ru brazing and W-CVD are not affected by high-temperature hydrogen, nitrogen, or helium. This paper details SC-Mo coaxial cavity STP thrusters made in three sizes — medium, small, and very small.

The thruster is supported by a pair of propellant supply pipes, which conduct part of the heat energy away from

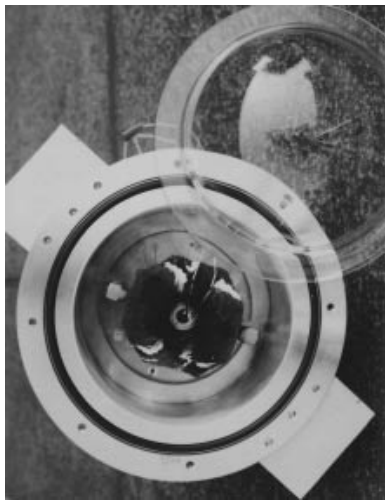

Fig. 2. The thruster, thermally insulated by carbon felt, is set in a vacuum chamber with a hemispherical Pyrex glass window through which concentrated solar rays are input, and the vacuum chamber is installed on a solar concentrator.

the thruster for regeneration with cold propellant gas. We measured the propellant gas temperature in the plenum and the surface temperature of the thruster outer wall using $\mathrm{Re}-5 \% \mathrm{~W} / \mathrm{Re}-26 \% \mathrm{~W}$ (up to $2,600 \mathrm{~K}$ ) thermocouples of $0.25 \mathrm{~mm}$ in diameter. Although multilayer insulation (MLI) has very high performance under vacuums such as those in space and much higher than in the vacuum chamber, we surrounded the thruster with carbon felt, instead of MLI, to insulate it thermally (Fig. 2). We confirmed that this insulation increased the gas temperature by about $400 \mathrm{~K} .{ }^{13)}$

The thruster was installed in a small vacuum chamber with a hemispherical Pyrex glass window through which concentrated solar rays were applied (Fig. 2). The total efficiency, including transparency and reflectivity, of this window was $80 \%$. The vacuum chamber was evacuated with a rotary pump and its pressure maintained at $0.3 \mathrm{kPa}$ while propellant nitrogen or helium gas was injected from the thruster nozzle at a 1 to 10 SLM propellant flow and a maximum plenum pressure of $0.2-0.4 \mathrm{MPa}$. Choke conditions were satisfied sufficiently in all experiments. The vacuum chamber containing the thruster was fixed on a $1.6 \mathrm{~m}$-diameter solar concentrator installed in a theodolite with a sun auto-tracking system at the NAL (Fig. 3) so that the thrust-
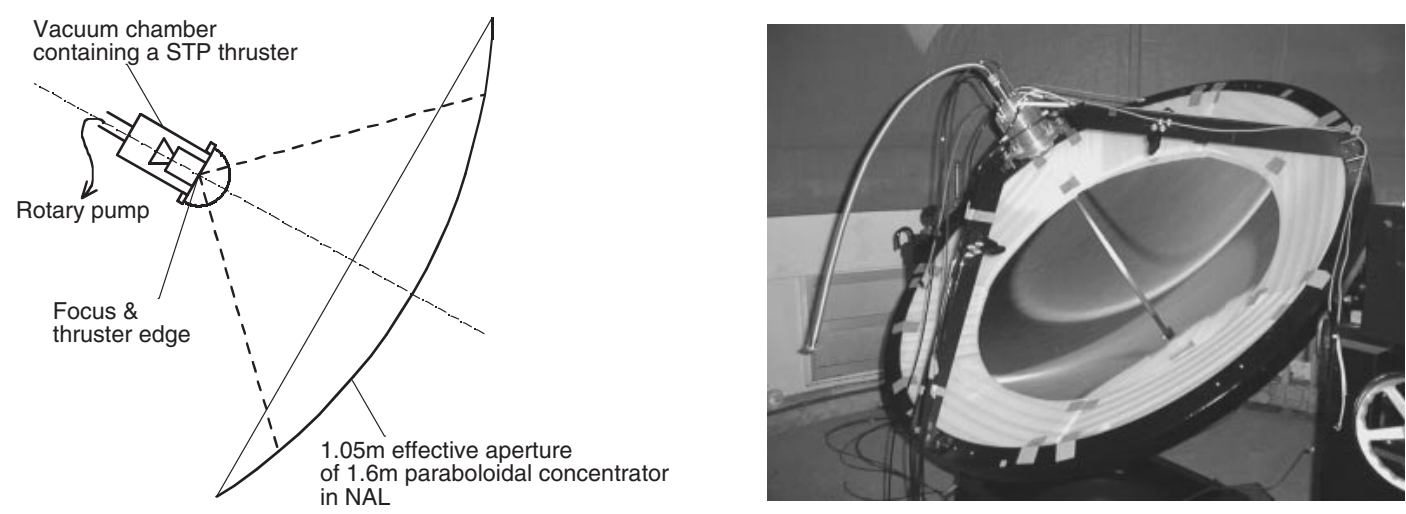

Fig. 3. The NAL solar concentrator and vacuum chamber at its focus, set in a sun autotracking system, is $1.6 \mathrm{~m}$ in diameter and has a focal length of $0.65 \mathrm{~m}$.

The thruster's cavity aperture is at the concentrator's focal point at the part of the concentrator that is $1.05 \mathrm{~m}$ in diameter, taking edge distortion into consideration. 


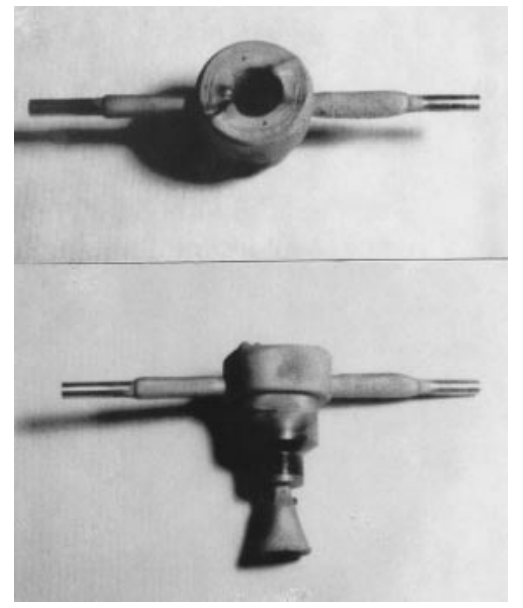

Fig. 4. The small NAL SC-Mo STP thruster with a W-CVD coating has a cavity diameter of $10 \mathrm{~mm}$, an outer diameter of $20 \mathrm{~mm}$, and is $36 \mathrm{~mm}$ long.

er's cavity aperture was at the focal point of the concentrator. Taking edge distortion into consideration, we used part of the concentrator, $1.05 \mathrm{~m}$ in diameter. Its focal length was $0.65 \mathrm{~m}$ and the ideal solar image diameter was $7 \mathrm{~mm}$ (specifically, about $10 \mathrm{~mm}$ ).

\subsection{Small STP thruster}

In the first stage of the STP thruster study using refractory metals, NAL designed, fabricated, and conducted experiments with a small SC-Mo thruster of $20 \mathrm{~mm}$ in outer diameter, $10 \mathrm{~mm}$ in cavity diameter, and $36 \mathrm{~mm}$ long (Fig. 4). This thruster is very suitable for the concentrator discussed above because of the good agreement between its cavity diameter and solar focal image diameter. In experimental results of heating and injection tests using nitrogen or helium as the propellant (Fig. 5), at the start of solar heating, the thruster temperature immediately rose and was saturated within 200 seconds. The propellant gas was fed through the supply pipes after the thruster temperature was almost saturated. Although it fell somewhat during propellant injection due to cooling by the propellant, thermal regeneration was possible. The higher the propellant flow, the lower the fall in temperature. Even so, thruster and propellant temperatures soon plateaued at 2,300 K for the propellant gas, $T_{\mathrm{g}}$, and $2,200 \mathrm{~K}$ for the thruster temperature, $T_{1}$, corresponding to an 800-second-class of $I_{s p}$ for the hydrogen propellant.

\subsection{Medium STP thruster}

Since good results were acquired by the small thruster, we enlarged the thruster. The medium SC-Mo thruster (Fig. 6) had an outer chamber of $30 \mathrm{~mm}$ in diameter including the W-CVD coating thickness, an inner chamber cavity of $20 \mathrm{~mm}$ in diameter, and was $61 \mathrm{~mm}$ long. This was a $2 \mathrm{~N}$ class thruster for hydrogen. The outer chamber size was within the upper limit of the SC-Mo cylinder diameter. The inner chamber was made by unifying the cylinder and bottom. NAL thus determined how to fabricate a thruster larger than SC-Mo bulk material dimensions by assembling thruster parts (i.e., the thruster's outer and inner chambers). In typical experimental results using a nitrogen gas propel-

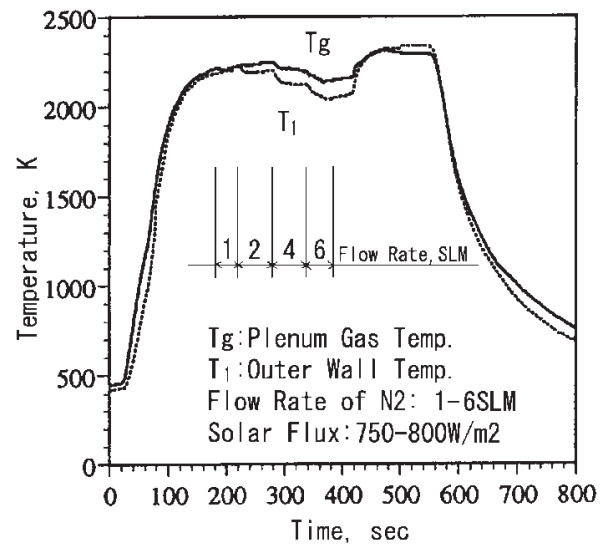

(a)

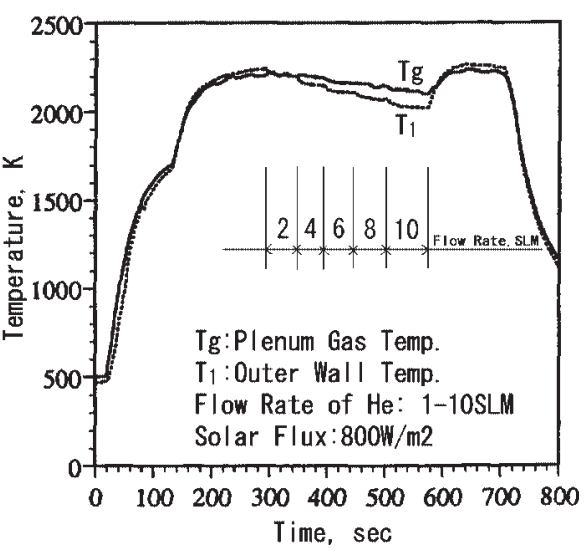

(b)

Fig. 5. Experimental results of heating and injection tests with the small NAL STP thruster using (a) nitrogen or (b) helium propellant. At the start of solar heating, the thruster temperature rose strongly and saturated within 200 seconds; the thruster was heated to $2,300 \mathrm{~K}$ and propellant to $2,200 \mathrm{~K}$.

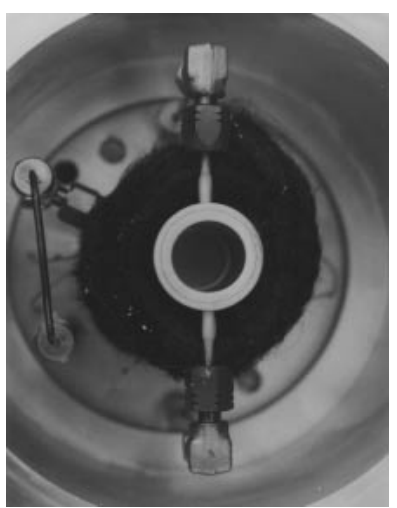

Fig. 6. The medium SC-Mo NAL STP thruster with a W-CVD coating has a cavity diameter of $20 \mathrm{~mm}$, an outer diameter of $30 \mathrm{~mm}$, and is $61 \mathrm{~mm}$ long.

lant (Fig. 7), propellant gas temperature $T_{\mathrm{g}}$ in the plenum reached about $1,600 \mathrm{~K}$ with the propellant flow, corresponding to about 650 seconds of $I_{s p}$ for hydrogen, while $T_{1}$ reached $1,800 \mathrm{~K}$. These temperatures are much lower than those of the small thruster detailed above because the larger outer surface results in greater heat loss. In short, the $1.05 \mathrm{~m}$ diameter concentrator was too small for the medium STP 


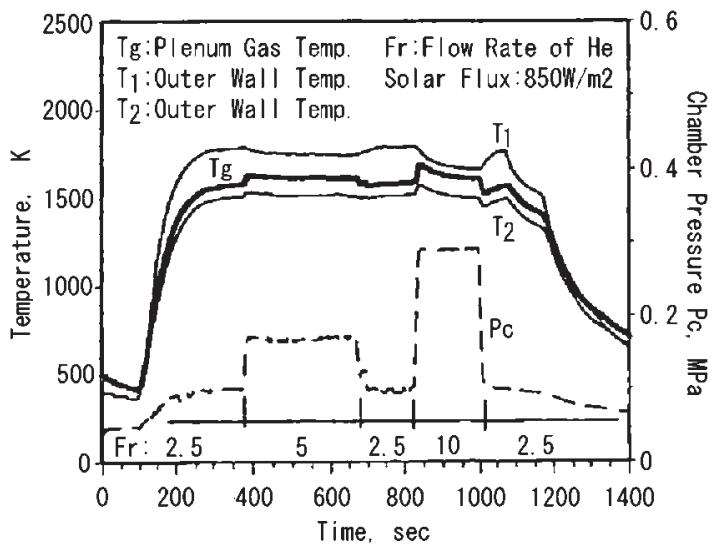

Fig. 7. Experimental results of heating and injection tests with the medium NAL STP thruster using nitrogen as the propellant.

Propellant gas temperature $T_{\mathrm{g}}$ in the plenum chamber reached about $1,600 \mathrm{~K}$ with the propellant flow.

thruster to ensure a higher propellant temperature.

\subsection{Very small STP thruster}

A very small SC-Mo $0.1 \mathrm{~N}$-class STP thruster (Fig. 8) was fabricated to determine manufacturing difficulty. The outer chamber of the thruster was $8 \mathrm{~mm}$ in diameter, the cavity $4 \mathrm{~mm}$ in diameter, and the thruster $23 \mathrm{~mm}$ long; the thruster weighed about 50 grams. The pair of propellant supply pipes was $3 \mathrm{~mm}$ in outer diameter and $2 \mathrm{~mm}$ in inner diameter. In heating and injection test results (Fig. 9), thruster tempera-

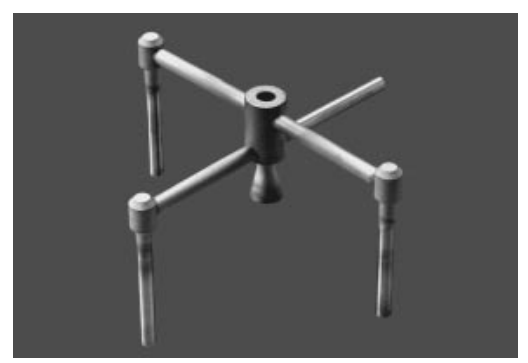

Fig. 8. The very small SC-Mo NAL STP thruster with a W-CVD coating has a cavity diameter of $4 \mathrm{~mm}$, an outer diameter of $8 \mathrm{~mm}$, and is $36 \mathrm{~mm}$ long.

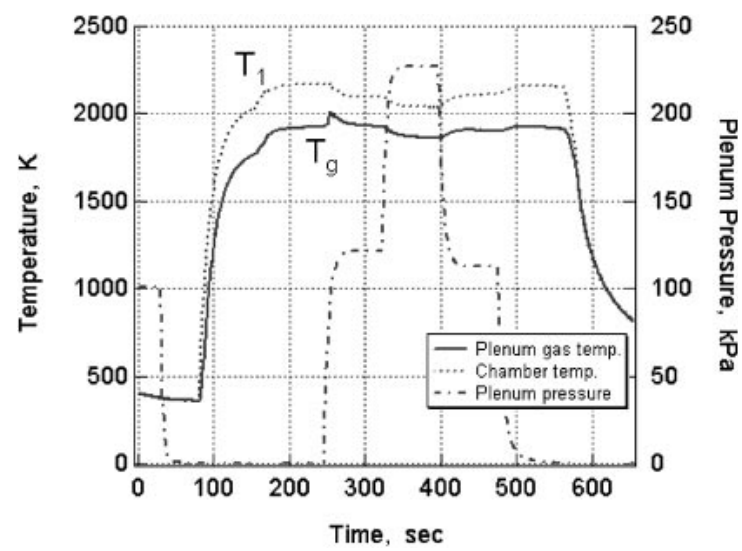

Fig. 9. Experimental results of heating and injection tests with the verysmall NAL STP thruster using nitrogen as the propellant. The thruster was heated to $2,200 \mathrm{~K}$ and propellant to $1,900 \mathrm{~K}$. ture $T_{1}$ was $2,100 \mathrm{~K}$ and propellant gas temperature $T_{\mathrm{g}}$ $1,900 \mathrm{~K}$ at a $1 \mathrm{SLM}$ nitrogen flow, corresponding to $750 \mathrm{sec}-$ onds of $I_{s p}$ for hydrogen. As the flow increased from 1 to 2 $\mathrm{SLM}, T_{\mathrm{g}}$ decreased inversely. The inner chamber cavity surface is ordinarily directly heated by concentrated solar rays, so the inner wall temperature is naturally highest, propellant temperature $T_{\mathrm{g}}$ second highest, and outer wall temperature $T_{1}$ the lowest. With the $1.05 \mathrm{~m}$-diameter concentrator used for the very small thruster, $T_{1}$ was much higher than $T_{\mathrm{g}}$. This inversion occurred because the solar image was much larger than the cavity, meaning that concentrated solar light also heated the thruster's outer wall much more than the inner cavity surface.

\section{Discussion}

The small STP thruster obtained very high temperature $2,300 \mathrm{~K}$ for the propellant gas and 2,200 K for the thruster's outer surface - at an appropriate propellant flow rate by solar heating and was equivalent to an 800-second-class STP thruster using hydrogen propellant. Since the melting point of Mo is $2,890 \mathrm{~K}$ and that of the W-CVD coating is $3,700 \mathrm{~K}$, the propellant gas temperature in the plenum remains highly reliable at about 2,500 K, corresponding to about $900 \mathrm{sec}-$ onds of $I_{s p}$ for hydrogen propellant. ${ }^{14)}$ The temperature obtained in experiments using the medium and very-small thrusters were, however, from 200 to $900 \mathrm{~K}$ lower than expected, primarily because, first, the $1.05 \mathrm{~m}$-diameter solar concentrator was too small for the medium thruster, which required a diameter of more than $1.5 \mathrm{~m}$ for the concentrator, and second, the propellant supply pipe diameter was large compared to that of the very small thruster, resulting in relatively large conduction heat loss through these pipes.

To confirm the above findings, we conducted a model calculation (ANSYS 5.7/6.0, Cybernet Systems Co., Ltd.). Alignment between the thruster and solar image focused by the concentrator depends on thruster and focal image diameter (Fig. 10). The cases of the medium, small and verysmall thrusters are represented by (a), (b), and (c) in the figure. Concentrated light thus was not always input to the entire cavity wall alone, which affects the temperature achieved by solar heating. In simulated results of experiments for the medium thruster (Fig. 11) and very-small thruster (Fig. 12), solar power density was $850 \mathrm{~W} / \mathrm{m}^{2}$, the focal image of the concentrator was $10 \mathrm{~mm}$ in diameter, and the heat transfer coefficient and other parameters were arranged to match results in the calculation. Heat conductivity, specific heat, and thermal radiation property depend on the material temperature. ${ }^{15-18)}$

For the medium thruster, $T_{1}$ and $T_{2}$ indicate temperatures simulated in the experiment with the $1.05 \mathrm{~m}$-diameter concentrator at the positions indicated in Fig. 11. The calculation result for the concentrator of $1.6 \mathrm{~m}$ in diameter (its focal image was $20 \mathrm{~mm}$ ) is indicated by $T_{1}{ }^{\mathrm{C}}$ and $T_{2}{ }^{\mathrm{C}}$ in the figure, and shows that the temperature rose $400 \mathrm{~K}$ due to the 2.3 fold increase in solar power heat. This means the thruster and propellant temperature will exceed $2,000 \mathrm{~K}$ using a geo- 

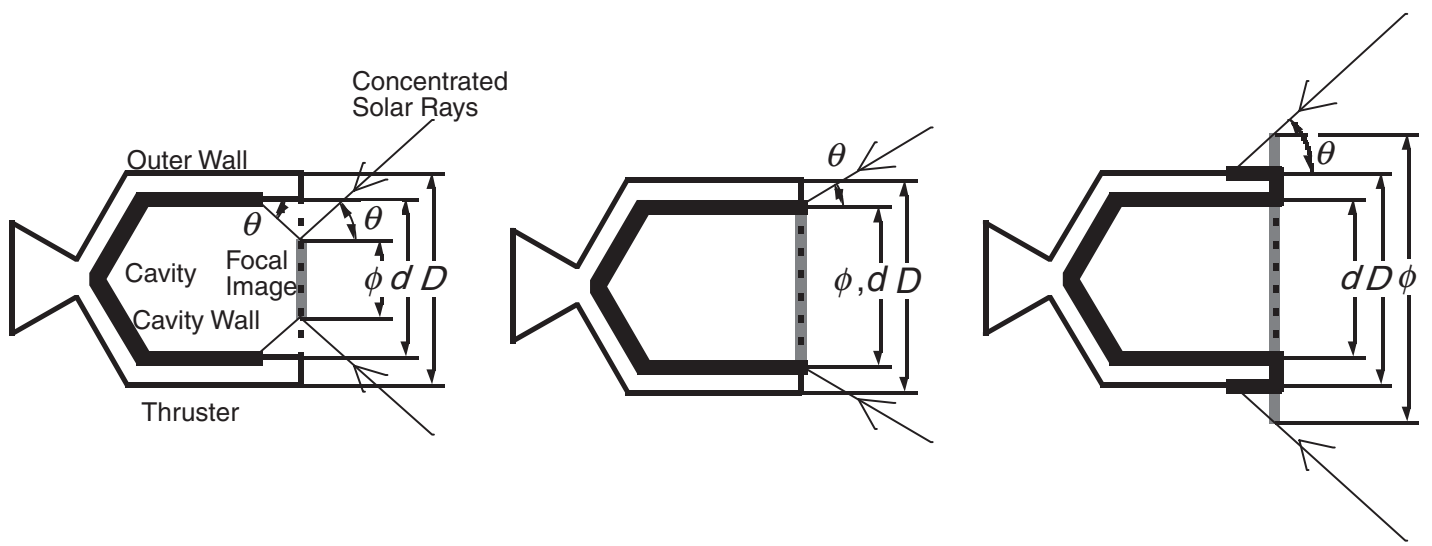

Fig. 10. Geometry of the thruster and concentrated solar rays.

$\mathrm{D}$ is the outer chamber diameter, $\mathrm{d}$ the cavity diameter, and phi the focal image diameter. Theta represents the value of focal length over diameter of the concentrator. The focal image diameter is (a) smaller, (b) equal to, and (c) larger than the cavity diameter.
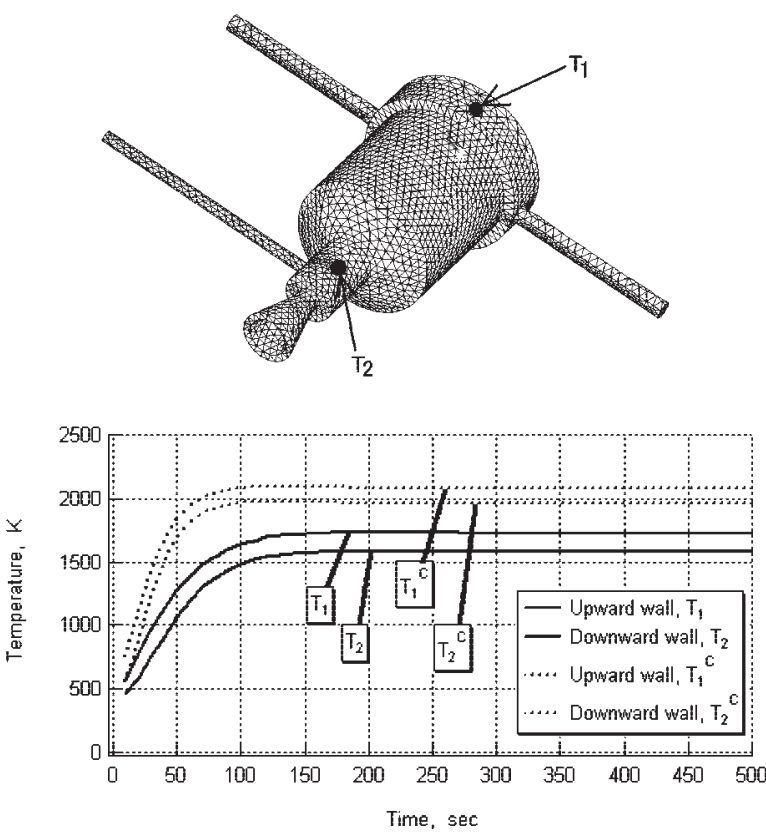

Fig. 11. Calculation result of thruster heating for the medium NAL STP thruster, showing the dependence of achieved temperature on input solar power.

The concentrator of solar power is (a) $1.05 \mathrm{~m}$ or (b) $1.6 \mathrm{~m}$ in diameter. Solar power density is $850 \mathrm{~W} / \mathrm{m}^{2}$ on the ground.

metrically optimized concentrator. On the other hand, the temperature simulated on the outer wall, $T_{1}$, using a propellant supply pipe of $3 \mathrm{~mm}$ in outer diameter is shown in Fig. 12 for the very-small thruster. The calculated result for a propellant supply pipe of $2 \mathrm{~mm}$ in outer diameter and having the same $1 \mathrm{~mm}$ inner diameter as the $3 \mathrm{~mm}$ pipe is indicated by $T_{1}{ }^{\mathrm{C}}$ in the figure. This indicates that a decrease in pipe diameter helped to raise thruster and propellant temperature because of decreased heat loss, but it is very difficult to machine these SC-Mo parts to a size much smaller than the current size, and the current manufacturing limit on outer pipe diameter exceeds $2 \mathrm{~mm}$. This is therefore a consideration requiring further study.

We obtained the best result for a propellant gas temperature of $2,300 \mathrm{~K}$ corresponding to 800 seconds of $I_{s p}$ for hy-
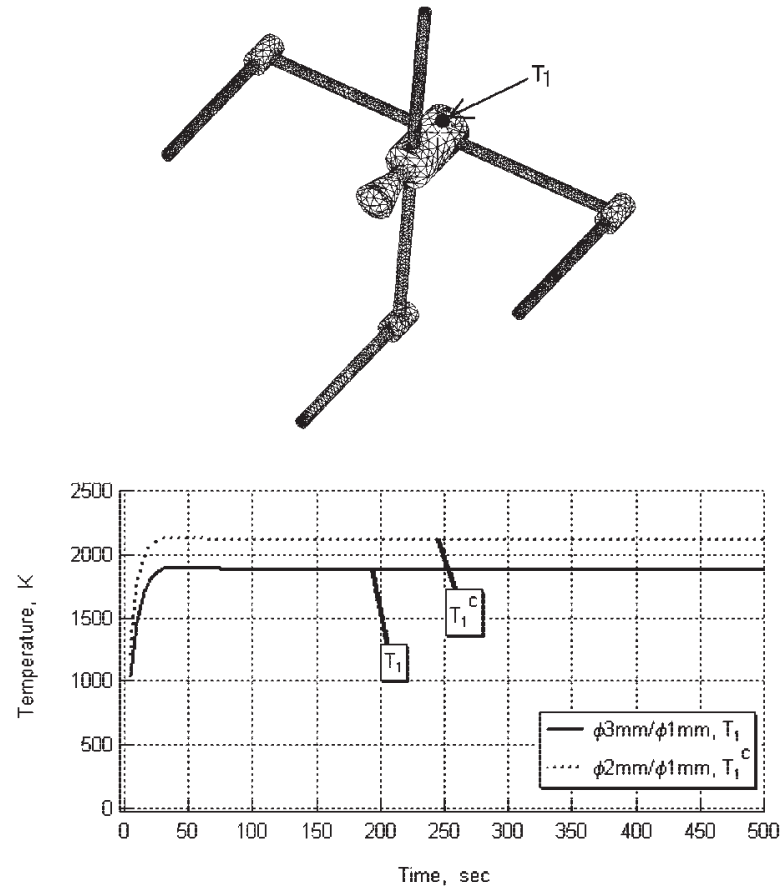

Fig. 12. Calculation result of thruster heating for the very-small NAL STP thruster, showing the dependence of achieved temperature on heat loss through propellant supply pipes.

Pipes are (a) $3 \mathrm{~mm}$ in outer and $1 \mathrm{~mm}$ in inner diameter, or (b) $2 \mathrm{~mm}$ in outer and $1 \mathrm{~mm}$ in inner diameter. Solar power density is $850 \mathrm{~W} / \mathrm{m}^{2}$ on the ground.

drogen in a ground test with the small thruster, but background vacuum conditions with and without propellant gas injection were only about $0.3 \mathrm{kPa}$. This means the heat loss by heat transfer to the ambient atmosphere was sufficient for the thruster temperature to become saturated at a lower temperature than expected. To evaluate the small STP thruster performance in space, we conducted a further model calculation, taking heat transfer and thermal radiation from the thruster surface into consideration to simulate experimental results in the calculation. Simulated temperature $T_{1}$ at a solar power input density of $850 \mathrm{~W} / \mathrm{m}^{2}$ (Fig. 13) agrees with the experimental result. To evaluate performance under the ultra-high vacuum of space, we changed the solar power 

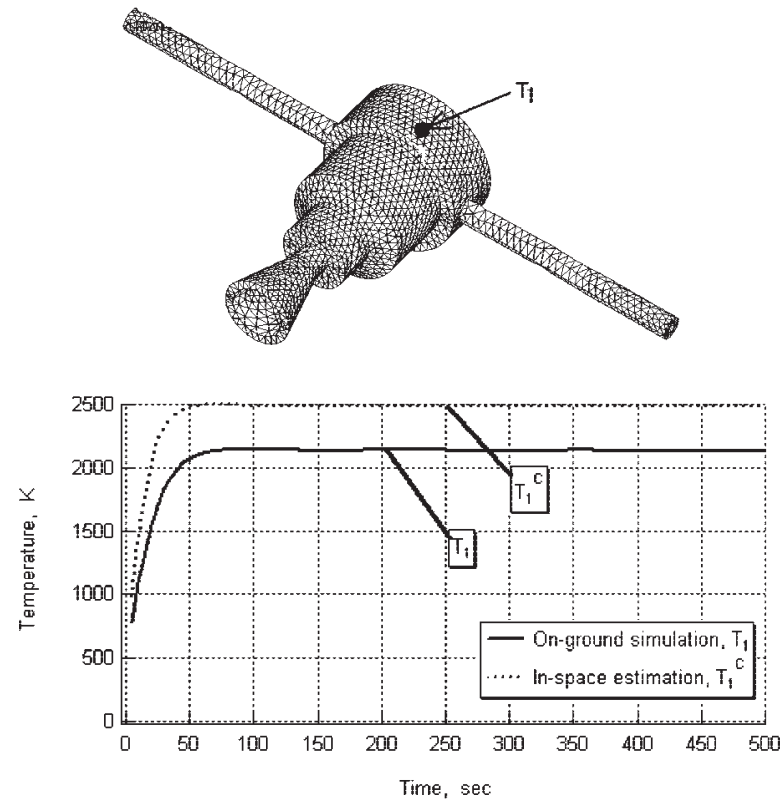

Fig. 13. Calculation results of thruster and propellant heating for the small NAL STP thruster used to evaluate its performance in space, for which experiments on the ground offer support.

(a) Solar power density is $850 \mathrm{~W} / \mathrm{m}^{2}$, hemispherical Pyrex glass window efficiency $80 \%$, and heat transfer on the thruster surface is included. (b) Solar power density is $1,400 \mathrm{~W} / \mathrm{m}^{2}$, no light is lost by the window, and no heat transfer occurs on the thruster surface.

input density to $1,400 \mathrm{~W} / \mathrm{m}^{2}$ and neglected heat transfer and light power loss of the Pyrex glass window. By nearly doubling the solar power input density and decreasing heat loss, we obtained $T_{1}{ }^{\mathrm{C}}$ (Fig. 13) indicating that the attained thruster temperature would be $2,500 \mathrm{~K}$ and the estimated propellant temperature would be about the same for our experimental results, meaning the thruster, with its 900 -secondclass thrust performance, would be one of the most suitable candidates for advanced propulsion in near-future space use.

\section{Summary}

We designed and fabricated STP thrusters of single-crystal molybdenum in three sizes - medium, small, and very small - to eliminate recrystallization embrittlement occurring at high temperature in a vacuum. We then conducted heating and injection tests using solar power concentrated by a concentrator of $1.05 \mathrm{~m}$ in diameter. With the small thruster, we attained a $2,300 \mathrm{~K}$ propellant gas temperature and 2,200 K thruster temperature; the thruster's target temperature was $2,000 \mathrm{~K}$ and its specific impulse 750 seconds. With the medium thruster, the concentrator was too small to obtain required the solar power, necessitating a concentrator of at least $1.6 \mathrm{~m}$ in diameter - a size shown by calculation to heat the thruster sufficiently. With the very small thruster, the comparably large diameter of the propellant supply pipes prevented the thruster from attaining a higher temperature due to heat loss by heat conduction. To solve this problem, propellant supply pipe diameter should be as small as possible, and we confirmed via calculation that this would decrease heat loss. To evaluate thruster performance in space, we conducted a model calculation for the small thruster and found that an 800 to 900 -second $I_{s p}$ with $0.1-$ $2 \mathrm{~N}$ thrust magnitude would be possible. Our STP technology and STP are thus viable for use in near-future propulsion such as that used in the upper stages of OTVs.

\section{References}

1) Shoji, J. M. and Frye, P. E.: Solar Thermal Propulsion for Orbit Transfer, AIAA Paper 88-3171, 1988.

2) McClanahan, J. A., Frye, P. E. and Borowski, S. K.: Evaluation of the Solar Thermal Propulsion Transfer Stage for Small Commercial Space Launch System Applications, AIAA Paper 94-3379, 1994.

3) Kennedy, C. F. and Jacox, M.: The Integrated Solar Upper Stage (ISUS) Program, AIAA Paper 95-3628, 1995.

4) Partch, R. and Frye, P. E.: Solar Orbit Transfer Vehicle Space Experiment Conceptual Design, AIAA Paper 99-2476, 1999.

5) Valentian, D., Amari, M., Fratacci, G. and Melchior, A.: A Comparison of Low Cost Cryogenic Propulsion and Solar Thermal Propulsion for Orbit Transfer, AIAA Paper 2002-3590, 2002.

6) Etheridge, F. G.: Solar-Rocket System Concept Analysis, AFRPL Tech. Rep., TR-79-79, 1979.

7) Shoji, J. M.: Solar Rocket Component Study, AFRPL Tech. Rep., TR84-057, 1985.

8) Kudija, C.: The Integrated Solar Upper Stage (ISUS) Engine Ground Demonstration (EGD), AIAA Paper 96-3043, 1996.

9) Lester, D. M., Wasson, S. R., Pearson, J. C. and Holmes, M. R.: Solar Thermal Propulsion IHPRPT Demonstration Program, AIAA Paper 2000-5109, 2000.

10) Suwama, Y., Takegahara, K., Funaki, I., Toki, K. and Shimizu, Y.: Preliminary Life Test of A Low-Power DC Arcjet Anode, 22nd ISTS, ISTS 2000-b-26, 2000.

11) Hiraoka, Y. and Fujii, T.: Welding and Joining of Single Crystals of BCC Refractory Metals, Proc. 12th Plansee Seminar in Austria, 1989.

12) Fujii, T.: A New Technique for Preparation of Large-Scaled Mo and W Single Crystals and Their Multilayer Crystals for Industrial Applications, Proc. Japan-Russia-Ukraine Intl. Workshop on Energy Conversion Materials (ENECOM 95), Sendai, 1995.

13) Shimizu, M., Itoh, K., Sato, H., Fujii, H. and Okamoto, K.: Fabrication and Tests of Single Crystal Mo Solar Thermal Thruster, 48th IAF, IAF-97-S.6.03, 1997.

14) Shimizu, M., Itoh, K., Sato, H., Fujii, T., Igarashi, T. and Okamoto, K.: Solar Thermal Thruster Made of Single Crystal Molybdenum, Acta Astronautica, 41 (1997), pp. 23-28.

15) Kotelinikov, R. B., Bashlikov, S. N., Galiakbarov, Z. G. and Kashtanov, A. I.: Super High-Melting Elements and Compounds, Endo, K. (ed.), Nisso Tsushinsha, Japan, 1977, p. 18.

16) The Japan Society of Mechanical Engineers: JSME Data Book: Heat Transfer, 4th ed., The Japan Society of Mechanical Engineers, Tokyo, 1986.

17) The Japan Society of Mechanical Engineers: JSME Data Book: Thermophysical Properties of Fluids, The Japan Society of Mechanical Engineers, Tokyo, 1986.

18) The Japan Society of Mechanical Engineers: JSME Heat Transfer Handbook, The Japan Society of Mechanical Engineers, Tokyo, 1993. 